\title{
Clarifications to the limitations of the $s-\alpha$ equilibrium model for gyrokinetic computations of turbulence
}

\author{
X. Lapillonne, ${ }^{1}$ S. Brunner, ${ }^{1}$ T. Dannert, ${ }^{1}$ S. Jolliet, ${ }^{1}$ A. Marinoni, ${ }^{1}$ L. Villard,${ }^{1}$ T. Görler, ${ }^{2}$ \\ F. Jenko, ${ }^{2}$ and F. Merz ${ }^{2}$ \\ ${ }^{1}$ Association Euratom-Confédération Suisse, Centre de Recherches en Physique des Plasmas, \\ Ecole Polytechnique Fédérale de Lausanne (EPFL), CH-1015 Lausanne, Switzerland \\ ${ }^{2}$ Max-Planck-Institut für Plasmaphysik, Boltzmannstr. 2, D-85748 Garching, Germany
}

(Received 24 July 2008; accepted 16 February 2009; published online 26 March 2009)

\begin{abstract}
In the context of gyrokinetic flux-tube simulations of microturbulence in magnetized toroidal plasmas, different treatments of the magnetic equilibrium are examined. Considering the Cyclone DIII-D base case parameter set [Dimits et al., Phys. Plasmas 7, 969 (2000)], significant differences in the linear growth rates, the linear and nonlinear critical temperature gradients, and the nonlinear ion heat diffusivities are observed between results obtained using either an $s$ - $\alpha$ or a magnetohydrodynamic (MHD) equilibrium. Similar disagreements have been reported previously [Redd et al., Phys. Plasmas 6, 1162 (1999)]. In this paper it is shown that these differences result primarily from the approximation made in the standard implementation of the $s$ - $\alpha$ model, in which the straight field line angle is identified to the poloidal angle, leading to inconsistencies of order $\varepsilon(\varepsilon=a / R$ is the inverse aspect ratio, $a$ the minor radius and $R$ the major radius). An equilibrium model with concentric, circular flux surfaces and a correct treatment of the straight field line angle gives results very close to those using a finite $\varepsilon$, low $\beta$ MHD equilibrium. Such detailed investigation of the equilibrium implementation is of particular interest when comparing flux tube and global codes. It is indeed shown here that previously reported agreements between local and global simulations in fact result from the order $\varepsilon$ inconsistencies in the $s$ - $\alpha$ model, coincidentally compensating finite $\rho^{*}$ effects in the global calculations, where $\rho^{*}=\rho_{s} / a$ with $\rho_{s}$ the ion sound Larmor radius. True convergence between local and global simulations is finally obtained by correct treatment of the geometry in both cases, and considering the appropriate $\rho^{*} \rightarrow 0$ limit in the latter case. (C) 2009 American Institute of Physics. [DOI: 10.1063/1.3096710]
\end{abstract}

\section{INTRODUCTION}

Among different potential technological alternatives for achieving thermonuclear fusion as an energy source, the tokamak, based on an axisymmetric magnetic confinement of a plasma, has been one of the most studied devices. In a fusion reactor, the energy and particle confinement times need to be long enough to obtain a positive energy balance between injected energy for heating the system and energy produced by fusion processes in the plasma. Energy and particle losses observed in magnetic fusion experiments are however well above those predicted considering purely collisional processes. This so-called anomalous transport is believed to result primarily from small scale instabilities, referred to as microinstabilities, which are driven by temperature and density gradients. Understanding microturbulence is therefore of major interest, as the associated transport, which directly depends on these gradients, determines the minimum size of a magnetic confinement-based fusion reactor. This microturbulence is widely studied via numerical simulations based on the nonlinear gyrokinetic equations. ${ }^{1-3}$

Since microinstabilities tend to have much longer wavelengths parallel than perpendicular to the equilibrium magnetic field, the computational effort can be significantly decreased by using a reduced simulation domain consisting of a narrow tube, elongated along a given field line, together with a field-aligned coordinate system. In such flux-tube simula- tions, ${ }^{4-7}$ a further local limit $\rho^{*}=\rho_{s} / a \rightarrow 0$, with $a$ the minor radius and $\rho_{s}$ the ion sound Larmor radius, is assumed and radial variations of density and temperature, as well as their gradients, are in particular neglected. This approach is to be distinguished from global simulations, where the full tokamak volume is considered and radial profiles of equilibrium quantities are taken into account.

In order to gain confidence in the turbulent transport level predicted by gyrokinetic simulations, benchmarking the various existing codes for defined sets of physical parameters is required. The Cyclone base parameters were derived from a DIII-D discharge in Ref. 5 and have been widely used in the past years for carrying out such validations and comparisons between different codes.

The aim of this work is to discuss and explain differences observed in gyrokinetic flux-tube computations when using different magnetic equilibrium models. We investigate in particular these differences for the Cyclone base case parameter set considering its importance as a benchmark. Furthermore, we address and clarify the fact that some previous comparisons between local and global simulations have been misinterpreted due to such equilibrium issues.

In Ref. 5, flux-tube results were obtained with various codes, all using the same reduced $s$ - $\alpha$ equilibrium model, ${ }^{8}$ which provides a simple, comprehensive, analytic model of essential magnetic equilibrium features and avoids having to 
interface with a magnetohydrodynamic (MHD) code. This model consists of circular shifted flux surfaces, with $s$ the shear and $\alpha$ the Shafranov shift between the centers of consecutive magnetic surfaces, resulting from the pressure gradient (note that for the Cyclone benchmark, low pressure is assumed and $\alpha$ is set to zero). However, it has already been pointed out previously that these simulations may differ strongly from results obtained using a more realistic magnetic equilibrium: In Ref. 9, both $s-\alpha$ and true MHD equilibria were considered, and significant differences in the corresponding linear gyrokinetic results were observed. These discrepancies were however assigned to the remaining Shafranov shift present in the low pressure MHD equilibrium plasma, which had been neglected in the $s$ - $\alpha$ model. Furthermore, it was also noted in Ref. 10 that results with an $s-\alpha$ model significantly differ from those obtained with a Miller ${ }^{11}$ geometry in the limit of circular parameters and no Shafranov shift, but no actual explanation was provided.

In this work, we show that the above mentioned differences in fact mainly result from the approximation made in the standard flux-tube implementation of the $s$ - $\alpha$ model, in which the straight field line poloidal angle (essential for a flux-tube model using a field-aligned coordinate system) is identified to the geometrical poloidal angle, which leads to inconsistencies of order $\varepsilon(\varepsilon=a / R$ is the inverse aspect ratio, $a$ the minor radius, and $R$ the major radius). Indeed, as shown in this paper, results with a low $\beta$, circular boundary MHD equilibrium are well recovered when using a model with circular, concentric magnetic flux surfaces which correctly treats the straight field line angle. Moreover, this model is straightforward to implement. Note that this rather technical aspect concerning order $\varepsilon$ inconsistencies is to be distinguished from physical finite $\varepsilon$ effects as those described in Ref. 12.

Considering the importance of the Cyclone case as a reference benchmark it appears essential to shed light on these geometry issues since they, in particular, lead to a coincidental agreement between flux-tube results with $s$ - $\alpha$ equilibrium and global results with a correct treatment of the equilibrium at finite $\rho^{*}=1 / 180$, as shall be clearly illustrated in this work. This particular value of $\rho^{*}$ is in fact consistent with the DIII-D discharge from which the Cyclone case is inspired. It is shown in this paper that a true agreement can be achieved when the flux tube and global simulations both correctly treat the equilibrium, and when the global computation is carried out for conditions approaching the $\rho^{*} \rightarrow 0$ limit, intrinsic to the flux-tube implementation.

The remainder of this paper, which can be viewed as a contribution to the current emphasis on validation and verification of fusion-relevant simulations, ${ }^{13}$ is organized as follows. In Sec. II, the gyrokinetic equations in general axisymmetric geometry, as implemented in the GENE (Refs. 6 and 14) code, are presented. In Sec. III, the different geometrical models which have been considered for the simulations are discussed: (1) an ideal MHD equilibrium provided by the CHEASE code, ${ }^{15}$ (2) an ad hoc, circular, concentric magnetic flux surface model which provides a simple correction to the flaws of the $s$ - $\alpha$ implementation, (3) a reminder of the standard $s$ - $\alpha$ implementation pointing out its order $\varepsilon$ inconsisten-
TABLE I. Normalization of independent variables.

\begin{tabular}{lccccc}
\hline \hline$\hat{t}$ & $\hat{x}$ & $\hat{y}$ & $\hat{z}$ & $\hat{v}_{\|}$ & $\hat{\mu}$ \\
\hline$c_{s} t / L_{\perp}$ & $x / \rho_{s}$ & $y / \rho_{s}$ & $z$ & $v_{\|} / v_{T i}$ & $\mu B_{0} / T_{i}$ \\
\hline \hline
\end{tabular}

cies. In Sec. IV, we present a detailed comparison between linear and nonlinear flux-tube simulations using the different geometrical models. These results are obtained with the GENE code, and confirmed in the linear regime by simulations carried out with the GS2 (Refs. 4 and 16) and GKW (Ref. 17) codes considering both $s-\alpha$ and MHD equilibria. In addition, simulations are carried out with GENE using the reduced ad hoc circular concentric model, which correctly treats the straight field line angle. The linear flux-tube results are then compared to global simulations using the GYGLES (Ref. 18) code, and agreement in the linear growth rate of ion temperature gradient (ITG) modes is shown when geometry is correctly implemented and in the appropriate limit $\rho^{*} \rightarrow 0$ for the global code. Conclusions are drawn in Sec. V.

\section{THE GYROKINETIC EQUATIONS FOR GENERAL AXISYMMETRIC EQUILIBRIA}

The simulations presented below are based on the Eulerian flux-tube code GENE, ${ }^{6,14}$ which solves the nonlinear, electromagnetic, multispecies, gyrokinetic equations on a fixed grid in phase space. In this paper, only electrostatic modes with one ion species and adiabatic electronic response are considered, and therefore the equations are expressed in this limit. We consider a Clebsch-type ${ }^{19}$ field-aligned $(x, y, z)$ coordinate system in which $(x, y)$ is the plane perpendicular to the magnetic field $\mathbf{B}=B_{0} \nabla x \times \nabla y, x$ is a magnetic flux surface label (radial coordinate in units of length), $y$ labels the field lines on a given flux surface (binormal coordinate in units of length), $z$ is an angle-like variable labeling the position along the field line (parallel coordinate), and $B_{0}$ is a reference magnetic field amplitude.

In the following, we thus present the electrostatic subset of the gyrokinetic equations as implemented in the GENE code, involving a number of terms which depend on the magnetic equilibrium. The ion particle distribution function $f_{i}$ is split into an equilibrium part $f_{0 i}$ and a small fluctuating part $f_{1 i}, f_{i}=f_{0 i}+f_{1 i}$, where

$$
f_{0 i}\left(v_{\|}, \mu\right)=\frac{n_{0}}{\left(2 \pi T_{i} / m_{i}\right)^{3 / 2}} \exp \left[-\frac{m_{i} v_{\|}^{2} / 2+\mu B}{T_{i}}\right]
$$

is a local Maxwellian with density $n_{0}$ and temperature $T_{i}$. The fluctuation $f_{1 i}$ depends on the $\left(x, y, z, v_{\|}, \mu\right)$ phase space coordinates and time $t$, where $v_{\|}$is the velocity parallel to the magnetic field, and $\mu=m_{i} v_{\perp}^{2} /(2 B)$ is the magnetic moment. The normalizations of the different quantities, chosen such that each normalized quantity is of order unity, are defined in Tables I and II, where $v_{T i}=\sqrt{2 T_{i} / m_{i}}$ and $c_{s}=\sqrt{Z_{i} T_{e} / m_{i}}$ are, respectively, the ion thermal and sound speed, $\rho_{s}=c_{s} / \Omega_{i}$ is the ion Larmor radius evaluated with the sound speed, and $L_{\perp}$ is a reference macroscopic length scale. In addition, gradients of equilibrium quantities are normalized to $L_{\perp}$ and one 
TABLE II. Normalization of dependent variables.

\begin{tabular}{lcc}
\hline \hline$\hat{f}_{j 0}$ & $\hat{f}_{j 1}$ & $\hat{\Phi}$ \\
\hline$f_{j 0} v_{T j}^{3} / n_{0}$ & $\left(f_{j 1} v_{T j}^{3} / n_{0}\right)\left(L_{\perp} / \rho_{s}\right)$ & $\left(e \Phi_{1} / T_{e}\right)\left(L_{\perp} / \rho_{s}\right)$ \\
\hline \hline
\end{tabular}

defines $\hat{B}=B / B_{0}$. Using this coordinate system and normalizations, the gyrokinetic equation for ions reads

$$
\begin{aligned}
\frac{\partial f_{1}}{\partial t}+ & {\left[\frac{1}{L_{n}}+\frac{1}{L_{T}}\left(v_{\|}^{2}+\mu B-3 / 2\right)\right] f_{0} \frac{\partial \bar{\Phi}_{1}}{\partial y} } \\
& +\left[\frac{\partial \bar{\Phi}_{1}}{\partial x} \frac{\partial f_{1}}{\partial y}-\frac{\partial \bar{\Phi}_{1}}{\partial y} \frac{\partial f_{1}}{\partial x}\right]+\frac{1}{B} \frac{\mu B+2 v_{\|}^{2}}{\sigma}\left(K_{x} \mathcal{G}_{x}+K_{y} \mathcal{G}_{y}\right) \\
& +\frac{v_{\|}^{2} \beta}{\sigma B} \frac{d p}{d x} \mathcal{G}_{y}+\alpha \frac{v_{\|}}{J B} \mathcal{G}_{z}-\frac{\mu \alpha}{2 J B} \frac{\partial f_{1}}{\partial v_{\|}} \frac{\partial B}{\partial z}=0 .
\end{aligned}
$$

As in Eq. (1), note that we shall drop the subscript labeling species-dependent variables as well as the hat for the normalized quantities so as to lighten notations. Here one has defined $\mathcal{G}_{j}=\partial_{j} f_{1}-\left(\sigma / v_{\|}\right) \partial_{j} \bar{\Phi}_{1} \partial f_{0} / \partial v_{\|}$for $j=(x, y, z), \alpha_{i}=v_{T i} / c_{s}$, and $\sigma_{i}=Z_{i} T_{e} / T_{i}$. The gyroaveraged electrostatic potential is denoted $\bar{\Phi}_{1}=J_{0}(\lambda) \Phi_{1}$, where $J_{0}$ is the zeroth-order Bessel function and $\lambda^{2}=k_{\perp}^{2}(2 \mu / B)$. The second term of Eq. (1) contains the equilibrium gradients driving term, with $1 / L_{T}$ $=-d \ln T / d x$ and $1 / L_{n}=-d \ln n / d x$, it is followed by the nonlinear $\mathbf{E} \times \mathbf{B}$ drift term. The term containing $K_{x}$ and $K_{y}$, as well as the following finite pressure term $(d p / d x$ is the radial derivative of the total plasma pressure) combine the magnetic curvature and $\nabla B$ drift effects. The coefficients $K_{x}$ and $K_{y}$ are given by

$$
\begin{aligned}
& K_{x}=-\frac{g^{x x} g^{y z}-g^{y x} g^{x z}}{B^{2}} \frac{\partial B}{\partial z}, \\
& K_{y}=\frac{\partial B}{\partial x}-\frac{g^{x y} g^{y z}-g^{y y} g^{x z}}{B^{2}} \frac{\partial B}{\partial z},
\end{aligned}
$$

with the metric tensor elements $g^{i j}=\nabla u^{i} \cdot \nabla u^{j}, u^{i}, u^{j}=x, y, z$. The pressure term is normalized to $\hat{p}=p / p_{\text {ref }}$, and $\beta$ is defined as $\beta=2 p_{\text {ref }} \mu_{0} / B_{0}^{2}$, where $p_{\text {ref }}$ is a reference pressure and $\mu_{0}$ is the vacuum permeability. The last two terms of Eq. (1) are related to particle trapping in the low magnetic field region of the magnetic confinement, and both include the Jacobian $J=J^{x y z}=[(\nabla x \times \nabla y) \cdot \nabla z]^{-1}$.

The self-consistent electrostatic potential $\Phi$ is obtained by solving the gyrokinetic quasineutrality equation (in normalized units)

$$
Z^{2} \tau\left[1-\Gamma_{0}(b)\right] \Phi_{1}=\pi Z B \int J_{0}(\lambda) f_{1} d v_{\|} d \mu-\left(\Phi_{1}-\left\langle\Phi_{1}\right\rangle\right),
$$

with $\tau=T_{e} / T_{i}, b=\left[1 /\left(Z_{i}^{2} \tau B^{2}\right)\right] k_{\perp}^{2}, k_{\perp}^{2}=g^{x x} k_{x}^{2}+g^{y y} k_{y}^{2}+g^{x y} k_{x} k_{y}$, $\Gamma_{0}(b)=\exp (-b) I_{0}(b)$ the scaled modified Bessel function, and $\left\langle\Phi_{1}\right\rangle$ the poloidal flux surface-averaged value of $\Phi_{1}$. The term on the left hand side of Eq. (2) is the polarization density, the first term on the right hand side is the perturbed ion gyrodensity, and the second term is the linearized adiabatic electron response.

The magnetic equilibrium thus enters the problem via the magnetic field amplitude $B(z)$, the Jacobian $J(z)$, the curvature terms $K_{x}(z)$ and $K_{y}(z)$, the pressure gradient $d p / d x$, and the metric tensor elements $g^{i j}(z)$ appearing in the quasineutrality equation through the perpendicular wave number $k_{\perp}$. Note that the pressure term is not taken into account in the following since low $\beta$ equilibria are considered.

\section{EQUILIBRIUM MODELS}

In this section, the different models which have been considered for describing the background magnetic equilibrium are presented.

\section{A. Interface with the MHD equilibrium code CHEASE}

One option in the GENE code is to obtain the relevant geometrical coefficients from realistic equilibria via an interface with the MHD equilibrium code CHEASE, ${ }^{15}$ which solves the Grad-Shafranov equation. CHEASE provides equilibrium quantities in the straight field line coordinate system $(\Psi, \chi, \phi)$, where $\Psi$ is the poloidal flux function, $\chi$ is the straight field line poloidal angle, and $\phi$ is the toroidal angle. These quantities are then transformed to the field-aligned coordinate system $(x, y, z)$ used in GENE according to the relations

$$
x=\frac{q_{0}}{r_{0} B_{0}} \Psi-x_{0}, \quad y=\frac{r_{0}}{q_{0}}(q \chi-\phi)-y_{0}, \quad z=\chi,
$$

where $r_{0}$ is taken here as the geometrical radius at the equatorial midplane of the magnetic surface considered in the flux-tube computation, $B_{0}$ is a reference magnetic field, $q_{0}$ is the safety factor at the considered flux surface, and $\left(x_{0}, y_{0}\right)$ define the center of the flux tube. The metric tensor in $(x, y, z)$ coordinates is expressed in terms of the metric tensor in $(\Psi, \chi, \phi)$ coordinates using the relations

$$
\begin{aligned}
& \nabla x=q_{0} /\left(r_{0} B_{0}\right) \nabla \Psi, \\
& \nabla y=\left(r_{0} / q_{0}\right)\left[q^{\prime} \chi \nabla \Psi+q \nabla \chi-\nabla \phi\right], \\
& \nabla z=\nabla \chi,
\end{aligned}
$$

which leads to

$$
\begin{aligned}
& g^{x x}=\left(\frac{q_{0}}{r_{0} B_{0}}\right)^{2} g^{\Psi \Psi}, \quad g^{x y}=\frac{1}{B_{0}}\left(q^{\prime} \chi g^{\Psi \Psi}+q g^{\Psi \chi}\right), \\
& g^{y y}=\left(\frac{r_{0}}{q_{0}}\right)^{2}\left[\left(q^{\prime}\right)^{2} \chi^{2} g^{\Psi \Psi}+2 q q^{\prime} \chi g^{\Psi \chi}+q^{2} g^{\chi \chi}+g^{\phi \phi}\right],
\end{aligned}
$$




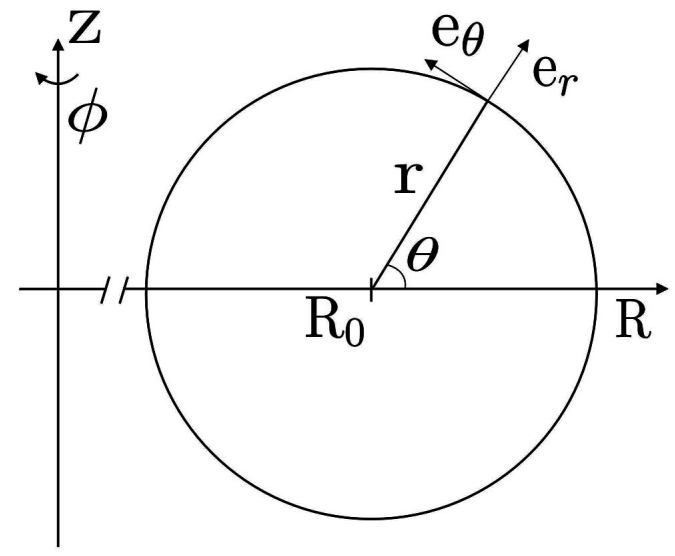

FIG. 1. Circular flux surface and $(r, \theta, \Phi)$ coordinates.

$$
\begin{aligned}
& g^{x z}=\frac{q_{0}}{r_{0} B_{0}} g^{\Psi \chi}, \quad g^{y z}=\frac{r_{0}}{q_{0}}\left(q^{\prime} \chi g^{\Psi \chi}+q g^{\chi \chi}\right), \\
& g^{z z}=g^{\chi \chi},
\end{aligned}
$$

with $q^{\prime}=d q / d \Psi$. The other required quantities are $\partial B / \partial x$ $=\left(r_{0} B_{0} / q_{0}\right) \partial B / \partial \Psi, \partial B / \partial z=\partial B / \partial \chi$, and $J^{x y z}=B_{0} J^{\Psi} \chi \Phi$, where $J^{a b c}=[(\nabla a \times \nabla b) \cdot \nabla c]^{-1}$. In the local approximation, which underlies flux-tube simulations, one neglects the $x$-dependence of all equilibrium quantities across the simulation domain. All geometrical coefficients therefore only need to be known on the magnetic surface of interest $\left(x=x_{0}\right)$, and are thus only functions of $z$ in the axisymmetric system considered here. Axisymmetry of the considered equilibria indeed translates into the independence with respect to $y$ of the coefficients. The CHEASE code has been modified to directly provide these coefficients on the z-grid required by GENE, i.e., an equidistant mesh along the straight field line angle $\chi$, and no further interpolation is required, thus taking advantage of the high precision cubic Hermite representation used in the equilibrium code. For the simulations in this paper, the MHD equilibria have been computed by prescribing the analytical shape of the last flux surface, together with pressure and current profiles. Note that the GENE interface with the CHEASE code is an alternative to its interface with the TRACER (Ref. 20) code to obtain geometrical coefficients for realistic geometry.

\section{B. Model with circular concentric flux surfaces}

In addition to the CHEASE interface, one also considers an ad hoc, analytical, axisymmetric, magnetic equilibrium model assuming circular, concentric flux surfaces. It is derived using the $(r, \theta, \phi)$ coordinates (see Fig. 1), where $(r, \theta)$ are poloidal coordinates in the $(R, Z)$ plane such that $R=R_{0}$ $+r \cos \theta$ and $Z=r \sin \theta, \phi$ is the toroidal angle, and $(R, Z, \phi)$ are cylindrical coordinates. The poloidal flux function $\Psi$ is prescribed to depend only on $r, \Psi=\Psi(r)$, and one assumes that $d \Psi / d r=r B_{0} / \bar{q}$, where $\bar{q}(r)$ is an ad hoc profile related to the true safety factor $q(r)$, as shown below in Eq. (6). The length $R_{0}$ thus appears as the major radius at the magnetic axis, i.e., at $r=0$. The toroidal magnetic component is prescribed to be given by $B_{\phi}=R_{0} B_{0} / R$, with $R=R_{0}(1+\epsilon \cos \theta)$, where $\epsilon=r / R_{0}$ is the inverse aspect ratio of a given magnetic surface. This leads to a magnetic field representation $\mathbf{B}=\nabla \phi \times \nabla \Psi+R B_{\phi} \nabla \phi=R_{0} B_{0} / R\left[\mathbf{e}_{\phi}+r /\left(R_{0} \bar{q}\right) \mathbf{e}_{\theta}\right]$, where $\mathbf{e}_{\phi}$ and $\mathbf{e}_{\theta}$ are the unit vectors along the toroidal and poloidal direction, respectively. The safety factor profile $q(r)$ is then given by

$$
q(r)=\frac{1}{2 \pi} \int_{0}^{2 \pi} \frac{\mathbf{B} \cdot \nabla \phi}{\mathbf{B} \cdot \nabla \theta} d \theta=\frac{\bar{q}(r)}{\sqrt{1-\epsilon^{2}}} .
$$

The straight field line angle $\chi$ is defined such that $(\mathbf{B} \cdot \nabla \phi) /(\mathbf{B} \cdot \nabla \chi)=q$, which leads to the relation $d \chi / d \theta$ $=\mathbf{B} \cdot \nabla \phi /(q \mathbf{B} \cdot \nabla \theta)$. Integrating over $\theta$ yields

$$
\chi(r, \theta)=\frac{1}{q} \int_{0}^{\theta} \frac{\mathbf{B} \cdot \nabla \phi}{\mathbf{B} \cdot \nabla \theta^{\prime}} d \theta^{\prime}=2 \arctan \left[\sqrt{\frac{1-\epsilon}{1+\epsilon}} \tan \left(\frac{\theta}{2}\right)\right] .
$$

This leads to the following metric tensor in $(\Psi, \chi, \phi)$ coordinates:

$$
\begin{aligned}
& g^{\Psi \Psi}=\frac{B_{0}^{2} r^{2}}{\bar{q}^{2}}, \quad g^{\chi \chi}=\frac{1}{r^{2}}\left[\frac{R_{0}^{2} \bar{q}^{2}}{R^{2} q^{2}}+\frac{\epsilon^{2} \sin ^{2} \chi}{\left(1-\epsilon^{2}\right)^{2}}\right], \\
& g^{\Psi \chi}=-\frac{B_{0} \epsilon}{\bar{q}} \frac{\sin \chi}{\left(1-\epsilon^{2}\right)}, \quad g^{\phi \phi}=\frac{1}{R^{2}}, \\
& g^{\Psi \phi}=g^{\chi \phi}=0 .
\end{aligned}
$$

The geometrical coefficients are then written in the $(x, y, z)$ coordinate system using Eqs. (5) and (8). Even though simulations presented in the following use the exact expressions, we explicit here the corresponding metric coefficients to first order in $\epsilon$,

$$
\begin{aligned}
& g^{x x}=1, \quad g^{x y}=\hat{s} \chi-\epsilon \sin \chi, \\
& g^{y y}=1+(\hat{s} \chi)^{2}-2 \epsilon \cos \chi-2 \hat{s} \chi \epsilon \sin \chi, \\
& g^{x z}=-\frac{\epsilon \sin \chi}{r_{0}}, \quad g^{y z}=\frac{1-2 \epsilon \cos \chi-\hat{s} \chi \epsilon \sin \chi}{r_{0}}, \\
& g^{z z}=\frac{1-2 \epsilon \cos \chi}{r_{0}^{2}} .
\end{aligned}
$$

where the magnetic shear is $\hat{s}=(r / q) d q / d r=\left(r^{2} B_{0} q^{\prime}\right) /(q \bar{q})$ and from Eq. (7) one has $\chi=\theta-\epsilon \sin \theta+\mathcal{O}\left(\epsilon^{2}\right)$.

\section{C. $s-\alpha$ model}

Finally, let us review the standard implementation of the so-called $s$ - $\alpha$ model for $\alpha=0$. One again considers a circular, concentric, magnetic surface geometry as in the previous model. The $(x, y, z)$ coordinates are defined as in Eq. (3) except for the straight field line angle $\chi$ which is approximated to the geometrical angle $\theta$ so that $y=\left(r_{0} / q_{0}\right)(q \theta-\phi)$ $-y_{0}$ and $z=\theta$. Despite this approximation, $(x, y, z)$ is still considered here as a field-aligned coordinate system. The metric coefficients to first order in $\epsilon$ are as follows: 


$$
\begin{aligned}
& g^{x x}=1, \quad g^{x y}=\hat{s} \theta, \quad g^{y y}=1+(\hat{s} \theta)^{2}, \quad g^{x z}=0, \\
& g^{y z}=1 / r_{0}, \quad g^{z z}=1 / r_{0}^{2} .
\end{aligned}
$$

A comparison with Eq. (9) exhibits differences of order $\epsilon$ between the two models, which points out that the approximation concerning the straight field line angle is only valid in the limit of an infinite aspect ratio tokamak $(\epsilon=0)$. Nevertheless, in order to retain trapping effects, the magnetic field amplitude is still defined for this model as

$$
\frac{B}{B_{0}} \simeq \frac{B_{\phi}}{B_{0}}=\frac{R_{0}}{R}=1 /(1+\epsilon \cos \theta),
$$

thus keeping finite aspect ratio terms. On the other hand, from the definition of the field-aligned coordinate system, one has $B=B_{0} \nabla x \times \nabla y$, which, from the metric coefficient (10) implies

$$
\begin{aligned}
\left(B / B_{0}\right)^{2} & =(\nabla x \times \nabla y)^{2}=(\nabla x)^{2}(\nabla y)^{2}-(\nabla x \cdot \nabla y)^{2} \\
& =g^{x x} g^{y y}-\left(g^{x y}\right)^{2}=1 .
\end{aligned}
$$

Comparing Eq. (11) with Eq. (12) underlines an inconsistency of order $\epsilon$ in the $s$ - $\alpha$ model, namely, the metric is computed as if $\epsilon=0$ but the magnetic field amplitude still retains an $\epsilon$ dependence. As will be shown in Sec. IV, this inconsistency leads, for finite $\epsilon$ cases, to significant differences between microturbulence simulations considering the $s$ - $\alpha$ model and simulations using either a MHD equilibrium or the previous concentric, circular model.

\section{CYCLONE TEST CASE}

In the following, Cyclone-like parameters ${ }^{5}$ are considered, namely, $n_{i} \equiv n_{e}, T_{e} / T_{i} \equiv 1, \quad q=1.42, \quad \hat{s}=(\rho / q) d q / d \rho$ $=0.8$, and $\epsilon_{0}=r_{0} / R_{0}=0.18$. Here, the normalized radial variable is defined for the MHD equilibrium as $\rho=\sqrt{\Phi / \Phi_{e}}$, $\Phi$ is the toroidal flux, and $\Phi_{e}$ is the value of the toroidal flux at the edge. For the ad hoc circular and $s$ - $\alpha$ models, one identifies $\rho=r / \alpha$. The gradient values are defined at $\rho=\rho_{0}$ $=0.5$ as $R_{0}\left\langle\nabla \ln T_{i}\right\rangle=6.96, \quad R_{0}\langle\nabla \ln n\rangle=2.23$, where $\langle A\rangle$ $=\int A J d \chi / \int J d \chi$ is the flux surface average of any quantity $A$. Note that the parameter $L_{T i}$ in Eq. (1) is estimated as follows:

$$
\begin{aligned}
\left\langle\nabla \ln T_{i}\right\rangle & =\langle|\nabla x|\rangle \frac{d \ln T_{i}}{d x}=-\frac{\langle|\nabla x|\rangle}{L_{T i}} \\
& \Rightarrow \frac{1}{L_{T i}}=-\frac{\left\langle\left|\nabla \ln T_{i}\right|\right\rangle}{\langle|\nabla x|\rangle},
\end{aligned}
$$

with a similar relation for $L_{n}$. We shall first focus on fluxtube computations and then compare these results to corresponding global ones.

\section{A. Linear flux-tube results}

Before considering nonlinear results, we first compare linear simulations using the three different equilibrium models described in Sec. III. Figures 2 and 3 show the linear growth rates and real frequencies of toroidal-ITG modes, respectively, as a function of the poloidal wave number $k_{y}$ in

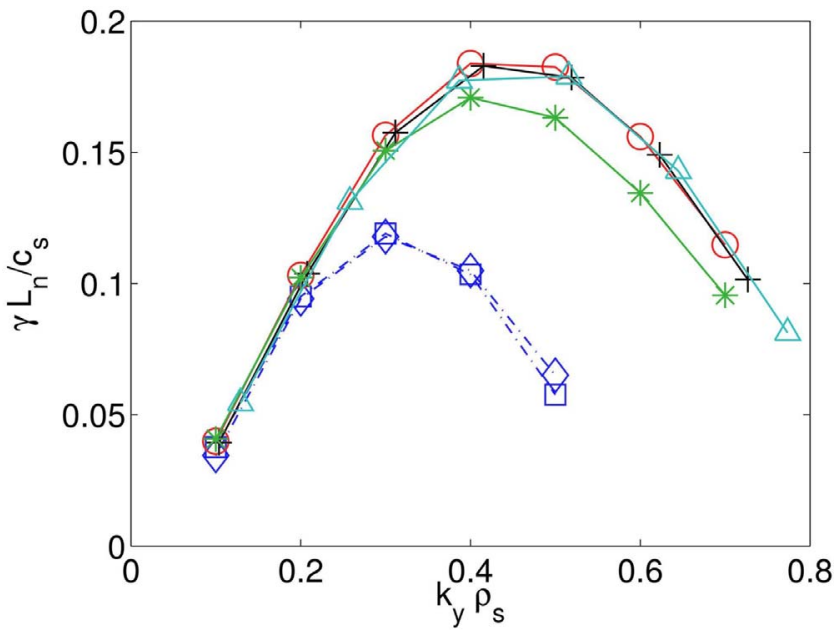

FIG. 2. (Color online) Growth rate as a function of the poloidal wave number $k_{y}$ of linear ITG modes for the Cyclone test case considering three different equilibrium models: MHD (circles: GENE; crosses: GS2; triangles: GKW), $s-\alpha$ (diamonds: GENE; squares: GS2), and ad hoc circular concentric (stars: GENE).

units of $\rho_{s}$, where $\rho_{s}$ has been evaluated with $T_{e}\left(\rho_{0}\right)$ and the magnetic field $B_{0}$ on axis. The results have been obtained from the flux-tube codes GENE, GS2, and GKW [formerly known as LINART (Ref. 17)] using an MHD equilibrium or the usual $s$ - $\alpha$ model, as well as from a GENE simulation using the corrected circular model. The MHD equilibrium used here is computed with the CHEASE code such that the last closed flux surface is circular and the Cyclone local parameters are matched at $\rho_{0}=0.5$. The pressure profile is chosen such that the value of $\beta=\langle p\rangle 2 \mu_{0} / B^{2}$ is small $\left(\sim 10^{-5}\right)$, where $\langle p\rangle$ is the volume-averaged pressure, and the current profile is adjusted so as to obtain the required value of the shear $\hat{s}$ at $\rho_{0}$. The growth rates and frequencies are of the order of the ion diamagnetic frequency which itself is of order $c_{s} / L_{\mathrm{ref}}$, where $L_{\text {ref }}$ is a characteristic gradient length of the system.

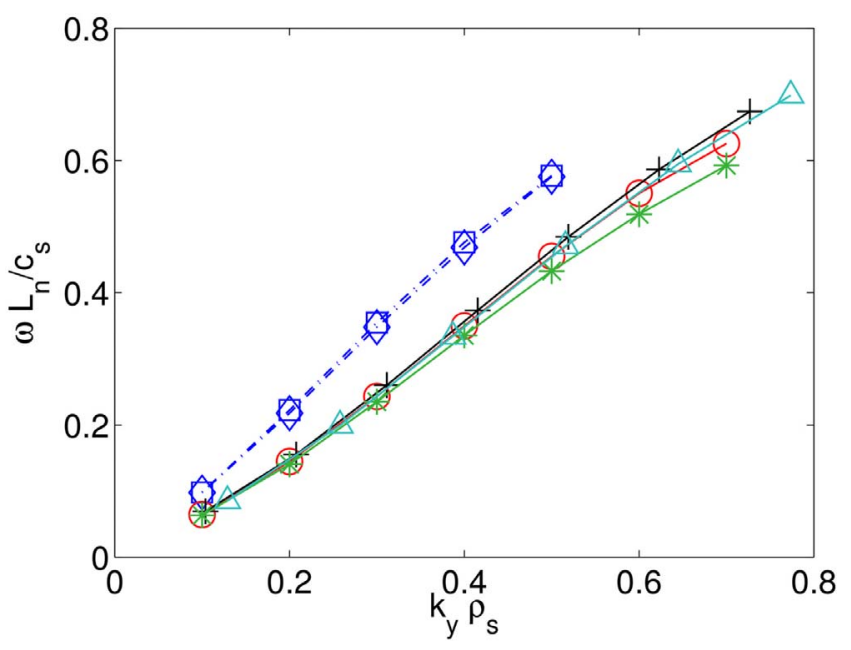

FIG. 3. (Color online) Real frequency spectra of linear ITG modes for the Cyclone test case considering different equilibrium models. Same labels as in Fig. 2. 

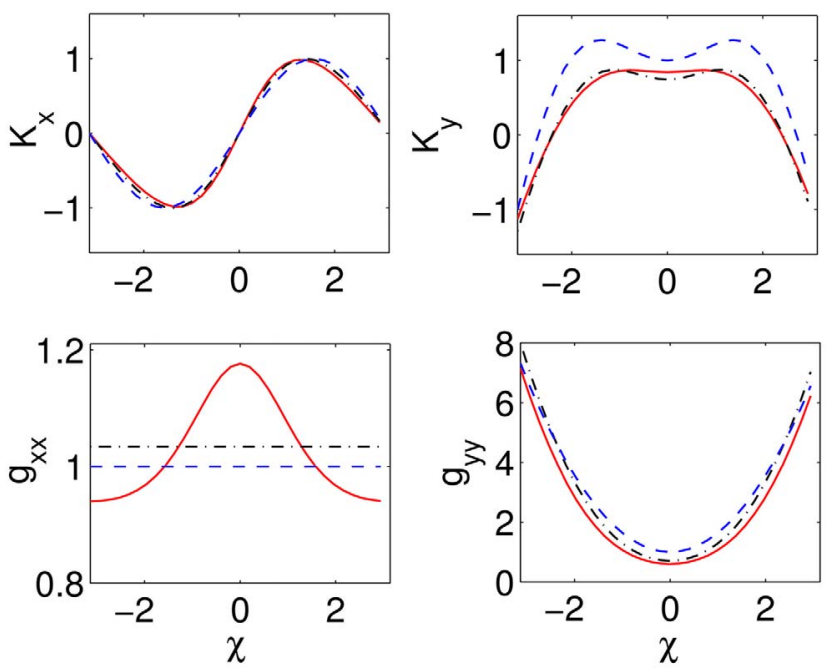

FIG. 4. (Color online) Geometrical coefficients for different equilibrium models: MHD (solid line), $s-\alpha$ (dashed line), and ad hoc circular concentric (dashed-dotted line).

Choosing $L_{\mathrm{ref}}=L_{n}$, frequencies and growth rates are thus normalized to $c_{s} / L_{n}$. This is the same normalization as considered in Ref. 5, which facilitates comparisons.

As clearly appears in Figs. 2 and 3, significant differences are observed, in agreement with Ref. 9, when comparing results using the reduced $s$ - $\alpha$ model or the MHD equilibrium and, in particular, the maximum linear growth rates differ by almost a factor of 2 . However, when using the analytical circular model, agreement with the MHD equilibrium case is reached within $10 \%$. This latter point clearly shows that the differences observed in this finite aspect ratio circular cross section geometry between simulations considering either the $s-\alpha$ or a realistic MHD equilibrium mainly result from the inconsistencies of order $\epsilon$ in the $s$ - $\alpha$ model pointed out in Sec. III C. By exchanging one geometrical term at a time in GENE's equations between the $s$ - $\alpha$ model and the circular analytic model, it can be shown that the differences result primarily from the discrepancies in the $g^{y y}$ and $K_{y}$ terms. In Fig. 4, the most relevant geometrical coefficients are plotted as a function of $\chi$ for the different equilibrium models described in Sec. III. The $g^{y y}$ and $K_{y}$ terms present the largest relative differences in the vicinity of $\chi$ $=0$ where ITG modes balloon. Furthermore, we note that the difference in the $g^{x x}$ term does not have a significant effect on the linear results, since it is the mode with $k_{x}=0$ which is the most unstable, corresponding to the perpendicular wave number $k_{\perp}^{2}=g^{y y} k_{y}^{2}$.

GENE simulations using the three different equilibria have also been compared for various values of the temperature gradient while keeping all other parameters as in the Cyclone test case. In Fig. 5, the maximum linear growth rate over all $k_{y}$ for ITG modes is given with respect to the normalized, flux surface-averaged temperature gradient $R_{0}\left\langle\nabla \ln T_{i}\right\rangle$. The linear critical temperature gradient obtained when using the $s-\alpha$ model is found around $R_{0}\left\langle\ln \nabla T_{i}\right\rangle=4$, which is in agreement with Ref. 5, and is decreased to $R_{0}\left\langle\ln T_{i}\right\rangle=3$ for realistic MHD equilibrium models as ob-

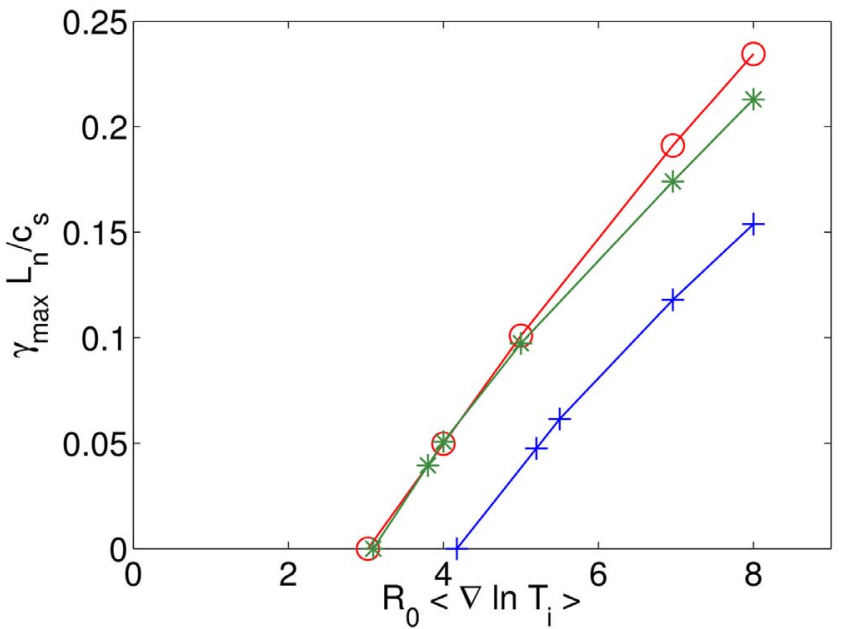

FIG. 5. (Color online) Growth rate of the most unstable ITG mode as a function of the ITG for MHD (circles), ad hoc circular concentric (stars), and $s$ - $\alpha$ (crosses) equilibrium models. All other parameters as in the $\mathrm{Cy}-$ clone test case.

served in Ref. 9. We note again that the MHD results are well recovered using the circular ad hoc equilibrium. This observation is of particular importance when using critical gradient values in semiempirical transport models. ${ }^{21}$

\section{B. Nonlinear flux-tube results}

As in the linear case, nonlinear simulations considering Cyclone test case parameters show strong discrepancies between results using $s-\alpha$ and MHD equilibria, while computations using the corrected circular model recover well those obtained with the MHD equilibrium.

Nonlinear simulations with the three different equilibria have been compared for Cyclone base parameters, with a numerical resolution $n_{x} \times n_{y} \times n_{z} \times n_{v \|} \times n_{\mu}=128 \times 48 \times 16$ $\times 32 \times 8$ and a perpendicular flux-tube box of dimensions $L_{x} \times L_{y}=118 \rho_{s} \times 96 \rho_{s}$. Figure 6 shows the ion heat diffusivity time trace. When using the MHD equilibrium, the time-

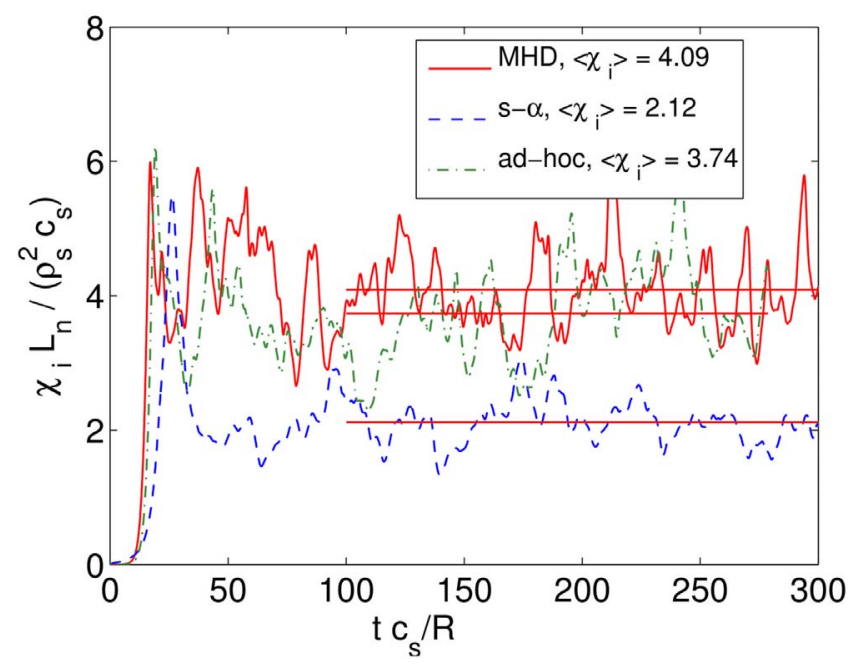

FIG. 6. (Color online) Ion heat diffusivity $\chi_{i}$ obtained by nonlinear GENE simulations for the Cyclone test case using MHD (solid line), $s-\alpha$ (dashed line) and ad hoc circular (dashed-dotted) equilibrium models. 


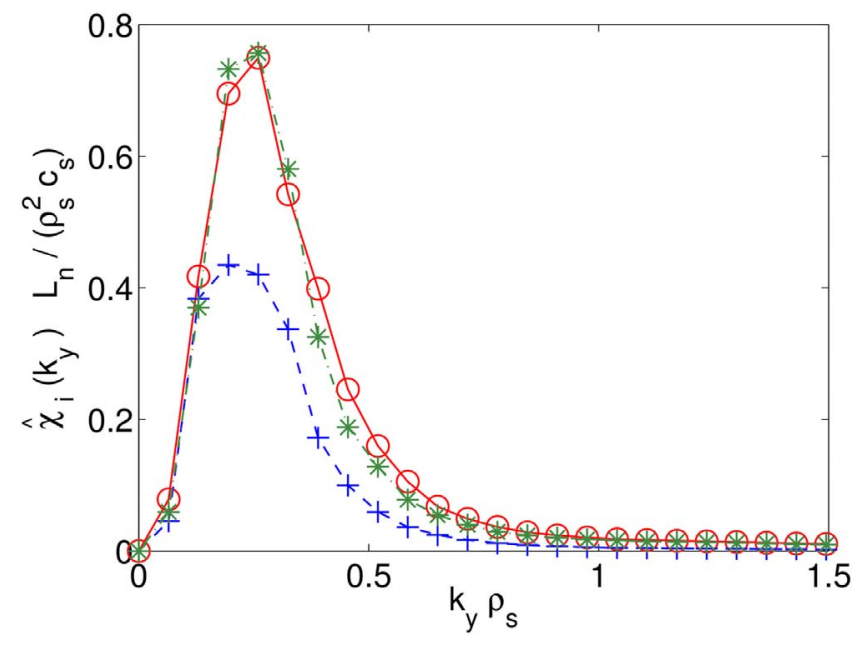

FIG. 7. (Color online) $k_{y}$ spectra of time-averaged ion heat diffusivity $\hat{\chi}_{i}\left(k_{y}\right)$ obtained via nonlinear GENE simulations for the Cyclone test case using MHD (circles), $s-\alpha$ (crosses), and ad hoc circular (stars) equilibrium models.

integrated ion heat diffusivity $\chi_{i}$ is estimated at $\chi_{i} L_{n} /\left(\rho_{s}^{2} c_{s}\right)$ $=4.1$ (corresponding to $\chi_{i} / \chi_{\mathrm{GB}}=3.3$, using the standard Gyro-Bohm normalization $\left.\chi_{\mathrm{GB}}=\rho_{s}^{2} c_{s} / a\right)$, which differs by almost a factor of 2 from the value obtained using the $s-\alpha$ model, for which $\chi_{i} L_{n} /\left(\rho_{s}^{2} c_{s}\right)=2.1\left(\chi_{i} / \chi_{\mathrm{GB}}=1.7\right)$. We note that the value $\chi_{i}$ for the $s$ - $\alpha$ case agrees with the LLNL GK fit

$$
\chi_{i} L_{n} /\left(\rho_{s}^{2} c_{s}\right)=15.4\left[1.0-6.0\left(L_{T} / R\right)\right]
$$

presented in Ref. 5, which also provides $\chi_{i} L_{n} /\left(\rho_{s}^{2} c_{s}\right)=2.1$ for $R / L_{T}=6.96$. The simulation using the ad hoc circular model gives $\chi_{i} L_{n} /\left(\rho_{s}^{2} c_{s}\right)=3.7\left(\chi_{i} / \chi_{\mathrm{GB}}=3.0\right)$ and thus agrees within $10 \%$ with the result using the MHD equilibrium. Strong discrepancies, for slightly different physical parameters, between nonlinear simulations using either the $s$ - $\alpha$ model or a corrected circular equilibrium obtained from the Miller model ${ }^{11}$ were also stated in Ref. 10, but no detailed explanation of the actual cause was provided.

In Fig. 7 the time-averaged ion heat diffusivity spectrum $\hat{\chi}_{i}\left(k_{y}\right)$, defined such that $\chi_{i}=\Sigma_{k_{y}} \hat{\chi}_{i}\left(k_{y}\right)$, is presented as a function of the normalized poloidal wave number $k_{y} \rho_{s}$. The spectrum obtained using the $s$ - $\alpha$ model strongly differs from the ones using either the ad hoc circular model or the MHD model, especially for $k_{y} \rho_{s}$ above 0.2 which corresponds to its maximum amplitude.

Figure 8 shows the time-averaged ion heat diffusivity using both the $s$ - $\alpha$ and MHD models for different values of the temperature gradient. The nonlinear critical gradients $R / L_{T, \text { crit }}$ are shifted upward with respect to the corresponding linear critical gradients according to the well known Dimits-shift ${ }^{5}$ effect. For the $s$ - $\alpha$ model, the resulting $R / L_{T \text { crit }}$ is around 6, identical to results in Ref. 5, and contained in the fit given by Eq. (14), while its value is around 5 using the MHD equilibrium.

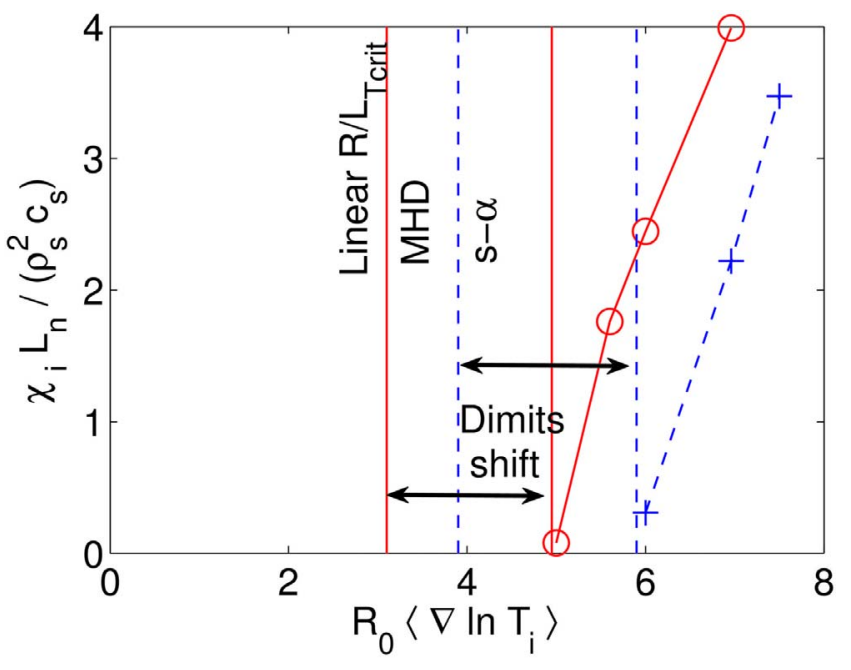

FIG. 8. (Color online) Ion heat diffusivity $\chi_{i}$ for the Cyclone test case as a function of the ITG. Results are given for simulations using either the MHD (circles) or the $s-\alpha$ (crosses) equilibrium model.

\section{Linear comparison with global simulations}

In view of the significant effects on the linear growth rates and nonlinear diffusivities from the approximations in implementing the $s$ - $\alpha$ model in the flux-tube simulations, the agreements between these same flux-tube results and global simulations reported in Ref. 5 appear surprising. Indeed, no similar approximations in implementing the equilibrium in the global simulations had been made. In order to address this apparent paradox, such local-global comparisons are repeated for linear simulations using GENE and the global, linear PIC code GYGLES. ${ }^{18}$ Global simulations, which consider the entire tokamak volume, are expected to approach the flux-tube results for $\rho^{*}=\rho_{s} / a \rightarrow 0$, where $a$ is the minor radius. Note that the limit $\rho^{*}=\rho_{s} / a \rightarrow 0$ is the assumption made in the standard flux-tube approach; this is in particular reflected by the fact that one neither accounts for the radial dependence of plasma density and temperature profiles and their gradients, nor for the radial dependence of magnetic equilibrium metric coefficients.

The GYGLES code is run using an analytical equilibrium with concentric, circular flux surfaces, identical to the one described previously in Sec. III B, with no further approximations on the geometry. The ad hoc safety factor profile is chosen to be $\bar{q}=0.854+2.184(r / a)^{2}$, corresponding to $\bar{q}\left(r_{0}\right)$ $=1.4$ and $\hat{\bar{s}}\left(r_{0}\right)=\left(r_{0} / \bar{q}\right) d \bar{q} / d r\left(r_{0}\right)=0.78$ at $r_{0} / a=0.5$ so that the values of the actual safety factor and shear assume the values of $q=\bar{q} /\left(1-\epsilon^{2}\right)^{1 / 2}=1.42$ and $\hat{s}=\hat{\bar{s}}+\epsilon^{2} /\left(1-\epsilon^{2}\right)=0.8$ used for the local simulations in the previous section. The temperature and density gradient profiles are defined as

$$
\begin{aligned}
& \frac{R_{0}}{T} \frac{d T}{d r}=-\kappa_{T} \cosh ^{-2}\left(\frac{r-r_{0}}{\Delta r}\right), \\
& \frac{R_{0}}{n} \frac{d n}{d r}=-\kappa_{n} \cosh ^{-2}\left(\frac{r-r_{0}}{\Delta r}\right),
\end{aligned}
$$

with peak gradients $\kappa_{T}=6.96, \kappa_{n}=2.23$ matching those con- 


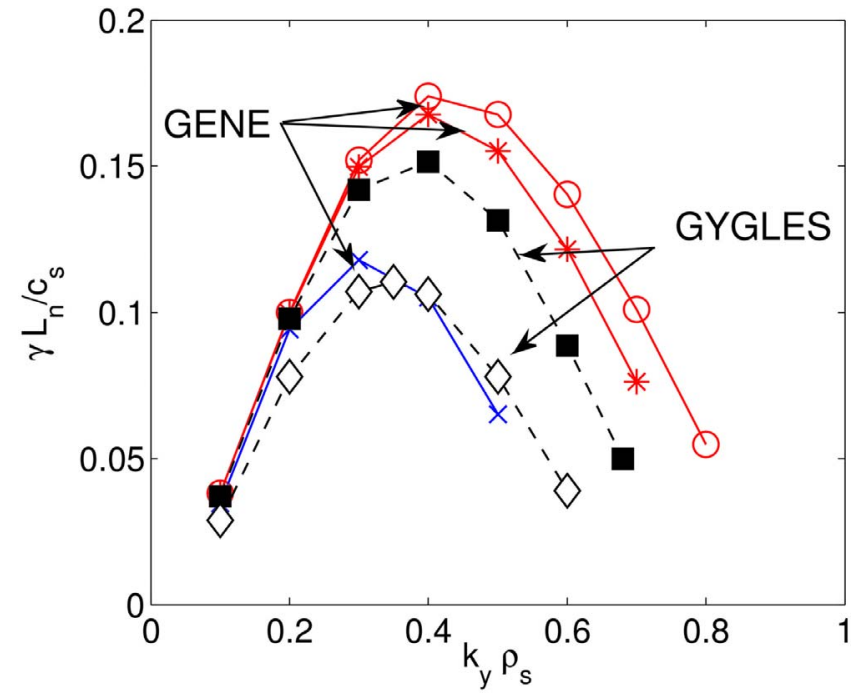

FIG. 9. (Color online) Growth rate spectra of linear ITG modes for the Cyclone test case as computed by the flux-tube code GENE and the global code GYGLES. GENE was run with either the $s$ - $\alpha$ (crosses) or circular analytical model, and for the latter considering the polarization drift term $\Gamma_{0}$ either to all orders in $k_{\perp} \rho_{s}$ (circles) or only to second order (stars). GYGLES results are given for $\rho^{*}=1 / 140$ (diamonds) and $\rho^{*}=1 / 1120$ (squares).

sidered in the GENE simulations and the radial width of the global profiles is chosen as $\Delta r=0.3 a$.

In Fig. 9, growth rates of ITG modes from GENE simulations using different geometrical models are plotted with respect to the poloidal wave number $k_{y} \rho_{s}$ and for GYGLES results for different values of $\rho^{*}$. The ratio $\rho^{*}$ is varied by rescaling the major and minor radii $R_{0}$ and $a$. For plotting results obtained from the global GYGLES code for a given toroidal wave number $n$, one makes use of the relation $k_{y}$ $=n q_{0} / r_{0}$. Indeed, using Eq. (3), one obtains

$$
\begin{aligned}
A(\Psi, \chi, \phi)=\hat{A}(\psi, \chi) e^{i n \phi} & =\hat{A}(\psi, \chi) e^{i\left[n q \chi-n q_{0} / r_{0}\left(y+y_{0}\right)\right]} \\
& =\hat{\mathcal{A}}(x, z) e^{-i k_{y} y} .
\end{aligned}
$$

A first series of global simulations with a value of $\rho^{*}$ $=1 / 140$ yields a $k_{y}$-spectrum which basically matches the flux-tube simulations using the $s$ - $\alpha$ geometry. A second $k_{y}$ spectrum obtained with the global code for the lower value $\rho^{*}=1 / 1120$ is however in closer agreement with the GENE results using the circular analytical model. Details of the convergence in $\rho^{*}$ of the GYGLES results going from $\rho^{*}$ $=1 / 70$ to $\rho^{*}=1 / 1120$ is shown in Fig. 10 for the fixed poloidal wave number $k_{y} \rho_{s}=0.3$. One notes that the apparent match, previously published in Ref. 5, between the global results for $1 / \rho^{*} \simeq 180$ (which is very close to the actual parameters of the DIII-D Tokamak from which the Cyclone case is inspired) and the flux-tube $s$ - $\alpha$ results is purely coincidental. However, when decreasing $\rho^{*}$ toward zero, the global simulation results truly converge toward the flux-tube results with correct treatment of the geometry, as clearly illustrated in Figs. 9 and 10.

In Fig. 9, one observes that there are nonetheless some remaining differences between the local and global results at the largest considered value $1 / \rho^{*}=1120$, which increase with $k_{y} \rho_{s}$. Part of this deviation is related to the different imple-

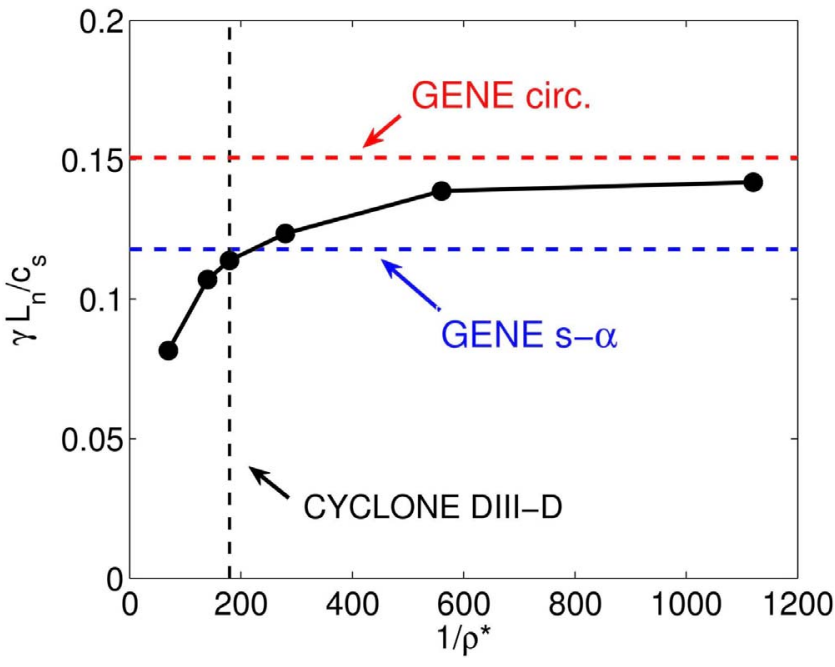

FIG. 10. (Color online) $\rho^{*}$ scaling of the ITG growth rate at $k_{y} \rho_{s}=0.3$ for the global code GYGLES. GENE results are obtained with the $s$ - $\alpha$ or the circular analytical equilibrium model.

mentations in the two codes of the quasineutrality equation. Indeed, taking advantage in the flux-tube calculation of the assumed periodicity in both the $x$ and $y$ directions, it is straightforward to express the polarization drift term in Fourier representation to all orders in $k_{\perp} \rho_{s}$. This term is represented in Eq. (2) by the modified Bessel function $\Gamma_{0}(b)$. On the other hand, in the global code GYGLES, the polarization drift term in the quasineutrality equation is handled in real space by a second order differential operator, corresponding in Fourier space to a second order Taylor expansion in $k_{\perp} \rho_{s}$ of $\Gamma_{0}(b)$. As shown in Fig. 8, a better agreement is thus obtained if one introduces the same approximation $\Gamma_{0}(b)$ $=1-b+\mathcal{O}\left(b^{2}\right) \simeq 1-k_{\perp}^{2} / \tau B^{2}$ in the flux-tube simulation. Note, that there still remain additional differences between the GENE and the GYGLES implementation of finite Larmor radius effects: In GYGLES the perturbed ion gyrodensity and the gyroaveraged potential $\bar{\Phi}_{1}$ are only evaluated up to second order in $k_{\perp} \rho_{s}$, whereas all orders are retained in GENE through the zeroth-order Bessel function $J_{0}(\lambda)$ appearing both in the relation $\bar{\Phi}_{1}=J_{0}(\lambda) \Phi_{1}$, as well as in the gyrodensity, i.e., the first term on the right hand side of Eq. (2).

\section{CONCLUSIONS}

In the present paper, we have investigated the influence of different treatments of the equilibrium in linear and nonlinear gyrokinetic simulations. The results presented here were focusing on the Cyclone DIII-D base case parameter set considering its importance as a reference benchmark. It has been shown that the significant differences obtained in linear and nonlinear simulations when using the $s$ - $\alpha$ model or a circular MHD equilibrium mainly result from approximating the poloidal angle to the straight field line angle in the standard implementation of the $s-\alpha$ model, which leads to inconsistencies of the order of the inverse aspect ratio $\varepsilon$. These differences reach a factor of almost 2 in the predicted turbulence induced heat flux for the standard Cyclone parameters. It is also found that using the $s-\alpha$ model the linear and non- 
linear critical gradients $R / L_{T, \text { crit }}$ are overestimated by at least $20 \%$. As a consequence, one should not only be aware of these discrepancies when comparing flux-tube codes among each other, but also when applying flux-tube results, and in particular the so-obtained critical gradients, to semiempirical transport models.

When considering a circular, concentric analytical equilibrium model which correctly treats the straight field line angle, one obtains good agreement with simulations using a true MHD equilibrium. This improved analytic model is applicable to both local and global simulations and is straightforward to implement in flux-tube codes.

The importance of using a correct geometrical model is of further interest when comparing results from flux-tube and global codes. In particular, it was shown in this paper that the previously reported apparent agreement between flux tube and global simulations ${ }^{5}$ had resulted from the unfortunate combination of two different effects, namely the inconsistencies of order $\varepsilon$ in the equilibrium model of the flux-tube codes and the physical finite size $\rho^{*}$ effects in the global simulations. True convergence between linear flux-tube results with a correct treatment of the geometry and global simulations in the appropriate limit of $\rho^{*} \rightarrow 0$ was demonstrated. In this respect, it is also interesting to note that the nonlinear $\rho^{*}$ scan in Ref. 22, using the global code GTC, converges to a value of $\chi_{i} / \chi_{\mathrm{GB}} \simeq 3.4$, which is in good agreement with our value of $\chi_{i} / \chi_{\mathrm{GB}} \simeq 3.0$ obtained with GENE using the corrected circular model. On the other hand, global GYRO results, in Ref. 23 converged toward the value of $\chi_{i} / \chi_{\mathrm{GB}} \simeq 1.9$ in the limit $\rho^{*} \rightarrow 0$, which is also in good agreement with the value of $\chi_{i} / \chi_{\mathrm{GB}} \simeq 2.1$ obtained with GENE using the $s-\alpha$ model.

\section{ACKNOWLEDGMENTS}

This work was partly supported by the Swiss National Science Foundation. The simulations have been run on the Pleiades cluster and the IBM Blue Gene/L parallel machine at EPFL. The authors would like to thank O. Sauter and T. M. Tran for their help concerning the implementation of the CHEASE code interface, as well as Y. Camenen for providing results from the GKW code.

${ }^{1}$ E. A. Frieman and L. Chen, Phys. Fluids 25, 502 (1982).

${ }^{2}$ T. S. Hahm, Phys. Fluids 31, 2670 (1988).

${ }^{3}$ A. J. Brizard and T. S. Hahm, Rev. Mod. Phys. 79, 421 (2007).

${ }^{4}$ M. Kotschenreuther, G. Rewoldt, and W. Tang, Comput. Phys. Commun. 88, 128 (1995).

${ }^{5}$ A. M. Dimits, G. Bateman, M. A. Beer, B. I. Cohen, W. Dorland, G. W. Hammett, C. Kim, J. E. Kinsey, M. Kotschenreuther, A. H. Kritz, L. L. Lao, J. Mandrekas, W. M. Nevins, S. E. Parker, A. J. Redd, D. E. Shumaker, R. Sydora, and J. Weiland, Phys. Plasmas 7, 969 (2000).

${ }^{6}$ F. Jenko, W. Dorland, M. Kotschenreuther, and B. N. Rogers, Phys. Plasmas 7, 1904 (2000).

${ }^{7}$ J. Candy and R. Waltz, J. Comput. Phys. 186, 545 (2003).

${ }^{8}$ J. W. Connor, R. J. Hastie, and J. B. Taylor, Phys. Rev. Lett. 40, 396 (1978).

${ }^{9}$ A. J. Redd, A. H. Kritz, G. Bateman, G. Rewoldt, and W. M. Tang, Phys. Plasmas 6, 1162 (1999).

${ }^{10}$ J. E. Kinsey, R. E. Waltz, and J. Candy, Phys. Plasmas 14, 102306 (2007).

${ }^{11}$ R. L. Miller, M. S. Chu, J. M. Greene, Y. R. Lin-Liu, and R. E. Waltz, Phys. Plasmas 5, 973 (1998).

${ }^{12}$ F. Jenko, W. Dorland, and G. W. Hammett, Phys. Plasmas 8, 4096 (2001).

${ }^{13}$ P. W. Terry, M. Greenwald, J.-N. Leboeuf, G. R. McKee, D. R. Mikkelsen, W. M. Nevins, D. E. Newman, D. P. Stotler, Task Group on Validation and Verification, U.S. Burning Plasma Organization, and U.S. Transport Task Force, Phys. Plasmas 15, 062503 (2008).

${ }^{14}$ T. Dannert and F. Jenko, Phys. Plasmas 12, 072309 (2005).

${ }^{15}$ H. Lütjens, A. Bondeson, and O. Sauter, Comput. Phys. Commun. 97, 219 (1996).

${ }^{16}$ W. Dorland, F. Jenko, M. Kotschenreuther, and B. N. Rogers, Phys. Rev. Lett. 85, 5579 (2000).

${ }^{17}$ A. Peeters and D. Strintzi, Phys. Plasmas 11, 3748 (2004).

${ }^{18}$ M. Fivaz, S. Brunner, G. de Ridder, O. Sauter, T. M. Tran, J. Vaclavik, L. Villard, and K. Appert, Comput. Phys. Commun. 111, 27 (1998).

${ }^{19}$ W. D. D'Haeseleer, W. N. G. Hitchon, J. D. Callen, and J. L. Shohet, Flux Coordinates and Magnetic Field Structure (Springer, Berlin, 1991).

${ }^{20}$ P. Xanthopoulos and F. Jenko, Phys. Plasmas 13, 092301 (2006).

${ }^{21}$ X. Garbet, Plasma Phys. Controlled Fusion 43, A251 (2001).

${ }^{22}$ Z. Lin, S. Ethier, T. S. Hahm, and W. M. Tang, Phys. Rev. Lett. 88, 195004 (2002)

${ }^{23}$ J. Candy, R. E. Waltz, and W. Dorland, Phys. Plasmas 11, L25 (2004). 